\title{
Tradiciones alimentarias y migración. Representaciones sobre la cocina coreana en Buenos Aires
}

\author{
Food traditions and migration. Representations about Korean \\ cuisine in Buenos Aires
}

Romina Delmonte rominadelmonte@gmail.com

http://orcid.org/0000-0001-8649-642X

Instituto de Investigaciones Gino Germani;

Facultad de Ciencias Sociales; Universidad de Buenos Aires/ Consejo Nacional de Investigaciones Científicas y Técnicas (Argentina)

\section{Resumen}

En este artículo proponemos una reflexión sobre las cocinas nacionales, las representaciones asociadas a ellas, y las formas en que estas son utilizadas en las construcciones identitarias en contextos migratorios. Específicamente analizamos el caso de los migrantes coreanos en la Ciudad de Buenos Aires, observando los sentidos que construyen en relación a la tradición alimentaria "coreana" y a la "argentina". Entendemos a las cocinas nacionales como construcciones históricas con cierto anclaje en la memoria colectiva y las prácticas pasadas, que se reconstruyen y reactualizan en cada contexto. Al igual que las identidades, las cocinas nacionales son relacionales, reconstruyen su sentidos en relación con un "otro". Así serán centrales tanto los procesos sociohistóricos que las fueron conformando, como los distintos discursos desplegados en políticas públicas, medios de comunicación y productos de la 
industria cultural. En un contexto migratorio como el que aquí analizamos, además, intervendrá el contacto intercultural que pondrá estas representaciones en diálogo, conflicto y tensión.

Palabras clave: Migración; alimentación; cocina; identidad; Corea.

\section{Abstract}

This article proposes a reflection on national cuisines, the representations associated with them and the ways in which they are used in the constructions of identities in migratory contexts. Specifically, we analyze the case of Korean migrants in Buenos Aires city, observing the senses associated with the "Korean" and "Argentinean" food traditions. National cuisines are historical constructions with certain anchoring in the collective memory and the past practices, which are reconstructed and updated in each context. We understand that, like identities, national cuisines are relational; they reconstruct their senses in relation to an "other". Thus, the sociohistorical processes that shaped them will be central, as well as the different discourses deployed in public policies, the media, and cultural industry products. And at the same time, in a migratory context like the one analyzed here, intercultural contact will intervene placing these representations in dialogue, conflict and tension.

Keywords: migration; food; cuisine; identity; Korea.

Los hábitos alimentarios de un grupo dan cuenta del pasado individual y colectivo, y a su vez están ligados a los sistemas de producción, consumo y comunicación de los que también son parte. La alimentación no se limita a la necesidad biológica de nutrirse, sino que conlleva implicancias económicas, sociales, ecológicas y culturales. Por lo tanto, "la práctica alimentaria es tributaria de la manera de pensar, de sentir y de actuar de los grupos étnicos" (Calvo, 1982: 385).

Barthes (2008) sostiene que los hábitos alimentarios significan, expresan y (re)crean una forma de vida y de entender el mundo. Por lo tanto, una cultura alimentaria es el resultado, nunca acabado, de la sedimentación de múltiples procesos: sociohistóricos, económicos, políticos, discursivos.

Desde nuestro enfoque entendemos que las formas de alimentarse de un grupo migrante se encuentran en sintonía con las configuraciones culturales (Grimson, 2011) presentes en el 
lugar de origen (en este caso la península coreana), y a su vez el proceso migratorio, con el contacto intercultural que trae aparejado, dará lugar a un proceso de reorganización de dichas prácticas.

Dentro de la gran multiplicidad de preguntas que podemos hacernos en relación a la alimentación de un grupo -cuestiones nutricionales, sobre el acceso a los alimentos, problemas relativos a la salud, sobre la soberanía alimentaria y la seguridad de los alimentos, cuestiones ligadas a lo económico y de estratificación o clases sociales, entre muchos otros- aquí nos preguntamos sobre el rol de la comida en la construcción de los sentidos de pertenencia, especialmente aquellos vinculados con lo nacional, en un contexto migratorio. Analizamos este problema ligado a cuestiones teóricas, en el contexto de la migración coreana en Buenos Aires en la actualidad. Nos preguntamos, ¿de qué modo construyen sentidos en relación a las cocinas nacionales? ¿Qué discursos los atraviesan? ¿De qué maneras la experiencia de ser migrantes en Buenos Aires reconstruye estas representaciones?

Entendemos a las identidades en tanto procesos múltiples, abiertos al cambio, atravesados por la dimensión del poder y por la historia, que se construyen a partir del discurso y del "hacer", que son relacionales y posicionales (Arfuch, 2002; Ricoeur, 2006[1990]; Hall, 2011; Grimson, 2011; Barth, 1976). Enfocándonos en el caso específico de las construcciones identitarias migrantes, consideramos a la migración coreana como diaspórica (Mera, 2007), por lo tanto la construcción de sus sentidos de pertenencia presenta ciertas particularidades, entre las que se destaca la articulación de representaciones sobre tres "espacios": el origen, otros lugares donde se asienta la diáspora coreana y el lugar de acogida (Argentina y especialmente Buenos Aires, en nuestro caso).

El trabajo de campo del que se desprende este artículo se desarrolló entre julio de 2014 y mayo de 2016. Se realizaron 26 entrevistas en profundidad a migrantes con una distribución homogénea según género y generaciones migrantes (1), de entre 20 y 78 años que viven actualmente en la Ciudad de Buenos Aires. De forma complementaria, y como parte de la investigación en la que se enmarca este trabajo (2), también se realizaron observaciones en templos religiosos, restaurantes, almacenes y eventos de la comunidad.

En cuanto a la organización de este artículo, comenzamos con una breve descripción de la instalación de los migrantes coreanos en la Ciudad de Buenos Aires. Continuamos con los aportes teóricos sobre las tradiciones alimentarias que delinean nuestro enfoque, luego describimos las políticas estatales en relación a la tradición alimentaria, desarrolladas por el gobierno de la República de Corea (3) en las últimas décadas. Por último, nos enfocamos en 
las representaciones de los migrantes coreanos sobre la cocina coreana y la cocina argentina, y cómo estas son utilizadas en sus construcciones identitarias.

\section{Breve descripción de la migración coreana en el país}

La migración coreana en la Argentina proviene mayoritariamente de Corea del Sur, aunque durante la década de 1950 llegaron unos pocos migrantes de Corea del Norte. Más allá de estos arribos más bien aislados, puede hablarse de una corriente migratoria a partir de 1965. A esta primera oleada migratoria, le siguen una segunda en la década de 1980 y la tercera y última en los noventa. En todos los casos se trata de una migración familiar. De este modo, en la actualidad la comunidad está conformada por tres generaciones migrantes.

Las particularidades y diferencias entre las tres oleadas migratorias radican, por un lado, en los cambios sufridos por la sociedad coreana, especialmente entre la década de 1960 y la de 1990, período en el que se produce un drástico proceso de industrialización y urbanización; así la segunda y tercera oleada contarán con mayor capital y nivel educativo que la primera. Y por otro lado, las diferencias también radican en la experiencia de la instalación en el nuevo destino. En el caso de los dos últimos grupos se encuentran con otros compatriotas ya asentados en el país, y redes de contención comunitaria. Pero así mismo, también se toparán con las representaciones que irá desplegando la sociedad mayoritaria sobre ellos, discursos en muchos casos xenófobos que estarán presentes en los medios de comunicación y distintos espacios de la vida cotidiana.

La comunidad coreana en Buenos Aires -en sintonía con su carácter diaspórico- concentra sus asociaciones, iglesias, negocios y en gran parte también viviendas, en dos barrios: el tradicional barrio coreano de Bajo Flores -Baek-ku (4)- y la zona de Floresta aledaña a la avenida Avellaneda -conocida como Ave entre los migrantes coreanos-. Ambos están localizados en la zona oeste de la Ciudad de Buenos Aires. Esta tendencia hacia la concentración territorial le permitió a la comunidad coreana construir un espacio propio, material y simbólico. Espacio que permite la re-creación de la identidad diaspórica (Santos Brightwell, 2012) como también de los valores y relaciones de poder de la diáspora. La concentración ayuda a articular una cierta idea de vida asociativa y comunitaria dentro del espacio urbano (Medina, 2008). En este marco, las diversas construcciones sobre la "cocina coreana" -que en Buenos Aires está prácticamente solo presente en los "barrios coreanos"colaborarán con la sensación de familiaridad, protección, el "sentirse en casa". 
En sintonía con la instalación concentrada en la ciudad, la comunidad coreana en Buenos Aires también se va a dedicar mayormente a la actividad textil, pequeñas y medianas industrias, y comercios mayoristas y minoristas. En la actualidad, la mayoría de estos comercios se ubican en la zona de la avenida Avellaneda.

\section{Una cocina nacional y las complejidades de su análisis}

Fischler (1995) sostiene que una cocina se inserta dentro de un "sistema culinario" regulado por ciertas reglas. De este modo, una cocina es un conjunto de ingredientes y de técnicas utilizadas en la preparación de la comida, junto con representaciones, creencias y prácticas que están asociadas a ella. Estas clasificaciones y reglas atienden tanto a la preparación y combinación de alimentos, como también a su producción y a su consumo. Así, una cocina no se define solo por un conjunto de alimentos, las formas de combinarlos y prepararlos, sino fundamentalmente por el conjunto del sistema culinario, que así como el lenguaje, es organizado por su propia gramática y sintaxis, lineamientos que le "dan sentido" y al mismo tiempo contribuyen a construir la familiaridad, es decir, la aceptabilidad de los platos (Fischler, 1995).

Por su lado, Sydney Mintz (1997) sostiene que no hay algo así como una cocina nacional, porque un estilo de cocinar y de comer no puede corresponderse con un límite político. De este modo, las cocinas son más bien regionales. En este sentido, Pettid (2008) señala que en el caso coreano la regionalización se va a ver profundizada por las características geográficas: el terreno montañoso fomentó la aparición y mantenimiento de las especialidades locales tanto en ingredientes y preparaciones como en métodos de procesamiento de los alimentos (2008: 24). Si no es la homogeneidad lo que define a la cocina tradicional coreana, ¿es lo tradicional lo que solo pertenece a los coreanos? Las teorías sobre globalización cobraron relevancia en las últimas décadas, pero la circulación de bienes y los intercambios culturales no son algo nuevo en la historia. En la cocina coreana encontramos múltiples influencias, incorporaciones e innovaciones que se incorporan como producto de acontecimientos políticos y sociales: desde la invasión mongola en el SXIII y el período de la dinastía Choson, hasta la ocupación japonesa del SXX. Por ejemplo, los mandu, considerados tradicionales de la cocina coreana, llegaron a Corea junto con los mongoles, como así también algunos platos a base de fideos de trigo y ciertas especias, como la pimienta negra (Pettid, 2008). Así mismo, aunque Corea tenga una larga historia en la realización de preparaciones fermentadas, el ají picante, ingrediente 
fundamental del kimchi (5) -el plato nacional en el que más adelante nos enfocaremos- tal cual existe hoy, llegó a la península desde América en el siglo XVI (Young, 2005). Pettid (2008) señala que durante la última mitad del período de la dinastía Choson, numerosos ingredientes de América se introducen a Corea a través de China, Japón, Europa y Filipinas, entre ellos: maíz, papas, batatas, ajíes, tomates, maní, calabaza y tabaco (2008: 18).

Encontramos en el concepto de "tradición inventada" de Hobsbawm algunos elementos que nos permiten echar luz sobre las tradiciones alimentarias y su relación con los sentidos y representaciones construidos en torno a lo nacional. "La 'tradición inventada' implica un grupo de prácticas, normalmente gobernadas por reglas aceptadas abierta 0 tácitamente y de naturaleza simbólica o ritual, que buscan inculcar determinados valores o normas de comportamiento por medio de su repetición" (2002: 8). Hobsbawm señala a la modernidad, y la conformación de los estados nacionales, como el proceso histórico que es condición de posibilidad y necesidad de estas tradiciones:

\footnotetext{
son muy importantes para la innovación histórica relativamente reciente que supone la "nación" y sus fenómenos asociados: el nacionalismo, la nación-estado, los símbolos nacionales, las historias y demás. Todo esto se basa en ejercicios de ingeniería social que a menudo son deliberados y siempre innovadores, aunque sólo sea porque la novedad histórica implica innovación (2002: 20).
}

Este concepto nos permite comprender que una cocina no se corresponde con un límite político de forma natural, por la mera existencia de esa frontera; pero, dentro de los mitos y relatos que construyen sentidos de pertenencia nacional, también se incluirán los que se relacionan con la alimentación. De este modo, al poner el acento en su carácter de construcción, no es nuestra intención menospreciar su influencia en las identidades, las prácticas cotidianas y las interacciones con los otros, sino más bien todo lo contrario. Es mediante este hacer que se construyen fronteras entre un "nosotros" y un "otros".

En un sentido similar, Grimson (2000) reflexiona sobre lo nacional, y con ecos de lo que más adelante plasmará con el concepto de configuraciones culturales (Grimson, 2011) sostiene que las "culturas nacionales" no existen como un conjunto objetivable de "rasgos típicos", sino más bien como campos de interlocución en los que algunas formas de identificación son legitimadas en los procesos de alianza y conflicto, mientras otras son invisibilizadas. En definitiva cada espacio nacional construye formas de articular y ocultar la diversidad, formas que así mismo no son estáticas sino que se reactualizan en los contextos específicos y como resultado de las luchas por el poder y la verdad. En este marco entendemos a las cocinas. Los hábitos y 
tradiciones alimentarias son parte de estos relatos que construyen sentidos de pertenencia, articulan heterogeneidades, diferencias y desigualdades, y por lo tanto definen lo nacional. Sin dejar de tener en cuenta que las cocinas nacionales son históricas, heterogéneas, relacionales, y cambiantes -no solo en cuanto a sus aspectos materiales, sino también en lo relativo a los sentidos que las atraviesan-, a continuación describiremos brevemente algunas particularidades de la cocina tradicional coreana, que creemos que delinean las prácticas y sentidos asociados a ellas, en el caso de los migrantes en Buenos Aires. Además, como veremos, presentan importantes diferencias con las tradiciones alimentarias preponderantes en la Ciudad de Buenos Aires, lo que habilita un encuentro intercultural signado por la diferencia. En primer lugar, los tres condimentos principales son ganjang (6), gochujang (7) y doenjang (8). Tres fermentos de la soja. Además, el conjunto de aromas de la condimentación de esta cocina se completa con aceite de sésamo, jengibre, ajo y ají picante (Pettid, 2008; Walraven, 2002). En cuanto a las preparaciones que conforman la mesa tradicional, del mismo modo que sucede con la cocina china, y a diferencia de las "cocinas occidentales", no hay un orden sucesivo de los platos, todo es servido y consumido en un mismo momento, de forma sincrónica. El arroz, que está presente en la mayoría de las comidas, es presentado en la mesa de forma individual y cada comensal tiene su propio bol. Por otro lado, se encuentra una sopa que también es dispuesta de forma individual en la mesa, a la derecha del arroz. Luego están los banchan similares al t'sai en la cocina china-, pequeños platos en base a carnes (de cerdo, pescado, vacuna), vegetales, hongos, algas, legumbres, preparados mediante distintos métodos de cocción (al vapor, salteados, hervidos, grillados, disecados, crudos, fermentados). Cada uno de los chop (unidades de banchan) idealmente no debe repetirse ni en cuanto a ingredientes ni en cuanto a métodos de cocción. Estas preparaciones son ubicadas en el centro de la mesa, de forma comunal, y cada uno de los comensales irá tomando su bocado. Dentro de los banchan, encontramos el kimchi, que como profundizaremos más adelante se destaca por su importancia simbólica del resto de las preparaciones. La mesa también puede incluir alguna preparación estofada -jjigae-.

Teniendo en cuenta estas características principales -y considerando que según el contexto se privilegiará el valor simbólico de uno u otro de estos aspectos-, a continuación nos concentramos en algunos procesos históricos atravesados por Corea en el último siglo, que entendemos que han delineado los sentidos y representaciones sobre esta cocina nacional. Especialmente nos enfocamos en las políticas públicas desarrolladas por el gobierno coreano a partir de la década de 1960, sobre todo en relación al kimchi, el plato icónico de la cocina coreana. 


\section{El kimchi como política de estado}

Desde un enfoque similar al de Hobsbawm, Young (2005) señala que las comidas nacionales emblemáticas, como el taco mexicano, la cerveza alemana, la pasta italiana y el sushi japonés, entre otras, son producto de fenómenos complejos en los que van a jugar un rol fundamental los movimientos nacionalistas modernos. En este sentido, la búsqueda de una cocina nacional comenzó en Corea en el pasado reciente, y el Estado ha jugado un rol activo en este proceso (Young, 2005; Osetrova, 2010; Pham, 2013).

Luego de décadas marcadas por procesos traumáticos como la ocupación japonesa y la guerra, a partir de la década de 1960 el gobierno coreano comenzará a promover la producción industrial de kimchi, a financiar investigaciones científicas en torno a la realización, guardado y empaquetado del kimchi y sus nutrientes, y a organizar acciones que difundan información sobre esta comida (Young, 2005). A partir de la década de 1980, Corea del Sur comienza a disfrutar los frutos del proceso de mejoras económicas, mediante el cual en pocos años pasa de ser un país predominantemente agrícola y arrasado por la guerra a uno fuertemente industrializado y urbano, aún en el marco de gobiernos autoritarios. En este contexto, en 1986 se inaugura el "Museo del kimchi" dedicado a difundir las características de esta preparación. Se llevan a cabo los Juegos Olímpicos en Seúl (1988) y se incluye el kimchi en la dieta de los deportistas (Osetrova, 2010). Tanto los Juegos Olímpicos como el Mundial Corea-Japón de 2002 fueron momentos que el gobierno utilizó para presentar ante la comunidad internacional, al kimchi como la comida nacional y representativa coreana. De este modo, este plato aparece asociado a la salud y la fuerza de los competidores coreanos. $Y$ así, se van a ir dejando atrás las representaciones de los años previos que ubicaban el consumo de kimchi como vergonzante -a causa de su olor fundamentalmente- ante los extranjeros (Cho, 2007). Entendemos que estos cambios se desarrollan en consonancia con determinadas condiciones históricas: el desarrollo económico ya consolidado, que venía gestándose desde las dos anteriores décadas; y el "orgullo nacional" asociado a este fenómeno que se expresaría en una valoración positiva de la tradición cultural (Ibídem: 213).

De aquí en más, el kimchi va a ir ganando mayor relevancia simbólica dentro de un discurso construido a través de políticas de gobierno: los textos escolares incluyen un apartado de comida, dándole especial importancia al kimchi, el almuerzo de alumnos y maestros brindado por las escuelas incluye kimchi todos los días (Young, 2005), se organizan "festivales de 
kimchi" patrocinados por el gobierno nacional. En el marco de este proceso, en la década de 1990 la industria alimentaria va a crecer en sintonía con el desarrollo del kimchi. En 1992, 160 empresas se dedican a la fabricación industrial de esta preparación, y en 2000 esa cifra asciende a 400; y así mismo en 1993, Samsung y LG lanzan las primeras heladeras diseñadas especialmente para kimchi (Cho, 2007).

A partir de la década del 2000, el Estado irá incluyendo la "cocina tradicional" -y especialmente el kimchi- crecientemente en su agenda de políticas públicas. En 2009 se crea el Comité de promoción de la Cocina Coreana (Hansik Development Globalization Agency), conformado por 36 miembros del gobierno, instituciones académicas y CEOs de la industria alimentaria coreana (Pham, 2013). Junto con el Ministerio de Comida, Agricultura, Forestación y Pesca presenta en 2010 el Hansik globalization Project (HGP), proyecto de globalización del hansik. El objetivo que declara públicamente es favorecer la imagen de (la marca) Corea a través de su cultura culinaria: exportación de alimentos coreanos y desarrollo de restaurantes en sincronía con la industria del turismo.

Este proyecto se lanza en el contexto del gobierno de Lee Myung-bak, ex CEO de Hyundai, quien asume en 2008 prometiendo la creación de un Consejo presidencial para la promoción de la "marca Corea". El HGP se va a articular en torno a discursos sobre la cocina coreana tradicional en los que se la representa, por un lado, como un producto cultural de identificación nacional, y por otro, como una cocina saludable, un "aporte de Corea al mundo" en términos de bienestar y salud. Este discurso buscará legitimación en el uso de datos estadísticos y un lenguaje científico. Los datos de referencia que presenta, son los resultados de estudios llevados a cabo por el mismo Ministerio de Comida, Agricultura, Forestación y Pesca en colaboración con universidades coreanas; que comprobarían los efectos beneficiosos de la comida coreana en distintos indicadores de salud, como los niveles de colesterol, e inclusive su impacto positivo en el tratamiento de enfermedades como diabetes y cáncer. Estas construcciones del gobierno coreano sobre la cocina nacional, que encontramos en las políticas de Lee Myung-bak, ubican a la comida como un producto cultural privilegiado en la construcción de la identidad, a la vez que se proponen proyectar sentidos en torno a lo coreano con el objetivo de desarrollar la "marca" Corea.

En el marco de este proceso, en 2013, la Organización de las Naciones Unidas para la Educación, la Ciencia y la Cultura (UNESCO) declara al kimchi y al kimjang (la práctica de preparar kimchi) de la República de Corea, como Patrimonio Cultural Inmaterial, alcanzando de este modo mayor proyección internacional y aún más legitimidad para ser incluido dentro de la agenda de políticas públicas del gobierno. El dictamen va a enfatizar los aspectos sociales y 
culturales involucrados en el kimjang y su carácter de práctica tradicional que contribuye a la construcción de sentidos de pertenencia. De este modo, se refuerza aún más la asociación de este plato con lo nacional.

El Hansik Globalization Project también desarrolla actividades en el exterior. Así llegó a Buenos Aires en julio de 2014 el kimchibus, un ómnibus que recorre ciudades del mundo, realizando demostraciones y degustaciones de comida coreana (kimchi, bibimbap, japchae, etcétera). Estas demostraciones son llevadas a cabo por jóvenes, con fuerte presencia en las redes sociales como Facebook, Instagram y Twitter. Estas estrategias se complementan, entre otras, con el lanzamiento en 2015 de guías de recetas de la cocina tradicional y de restaurantes coreanos en distintas ciudades del mundo, en forma de "aplicaciones" para smartphones.

Por otro lado, también es interesante para profundizar en otros trabajos, la relación entre estas políticas de "globalización del hansik" y el hallyu, la "ola coreana" -la difusión a partir de mediados de la década de 1990 de productos culturales como las telenovelas y la música pop-. Ambos fenómenos ocurren en el mismo período de tiempo, pueden incluirse dentro del concepto de softpower, $y$ como señala Ongkowidjojo (2015) ambas tendencias se potencian, mientras los cantantes de k-pop son utilizados para promover el hansik, la cocina también es utilizada como puerta de entrada para el consumo de otros productos culturales.

\section{Los migrantes y la cocina coreana en Buenos Aires}

Como señalamos anteriormente, entendemos que una forma de comer no se corresponde de forma natural con un Estado o un límite político. Hay muchas formas de "comer coreano" y de "comer argentino", o más bien, las cocinas nacionales se conforman de heterogeneidades articuladas y negociadas en los contextos socio-históricos (Delmonte, 2016; 2017).

Más allá de las complejidades de pensar una cocina, sus límites, sus desigualdades, sus alcances, para los migrantes coreanos entrevistados había algo que era "comida coreana" y otra cosa -distinta, en algunos casos, opuesta- que era "comida argentina", categorías que además organizaban las prácticas, y como veremos eran utilizadas en la construcción de identidades. Ante estas formas de nombrar, aparecieron las preguntas. ¿Qué condiciones llevan a que ciertas preparaciones y formas de cocinar sean colocadas bajo una misma etiqueta, un nombre? ¿Qué sentidos encierran para los migrantes entrevistados el "comer coreano" o "comer argentino"? 
Janowski señala que aunque la convivencia de fuerzas de continuidad y cambio sea una característica general de los hábitos alimentarios en los distintos grupos sociales, en el caso de las comunidades migrantes el cambio ocurre de distintas maneras que en el país de origen y, asimismo, en este contexto, la "idea" de una forma de comer puede verse "fosilizada" (2013: 176). De este modo, podemos interpretar cómo algunos elementos de la tradición alimentaria van a ser idealizados. En un sentido similar, sostiene que en contextos de migración o desplazamiento existe una tendencia a utopizar los recuerdos, prácticas y productos asociados al país de origen, en lo que denomina "ideación" del pasado. Grimson (2011) por su parte, sostiene que aunque "cultura" e identidad no siempre coinciden, en el contexto migratorio algunos elementos de la trama cultural son tomados, utilizados y proyectados en relación con procesos identitarios. Este es el caso de ciertos alimentos y prácticas relacionados con la comida para la comunidad coreana en Buenos Aires.

En el contexto migratorio, el referente nacional -"comida coreana"- acentúa su importancia simbólica, disminuyendo la de otros anclajes identitarios, como los regionales, generacionales, de clase, de género, etcétera. Categorías que diferencian las prácticas alimentarias, los sentidos asociados y los usos en las construcciones identitarias.

Así, en este contexto la "comida coreana" es entendida como diferente, e incluso opuesta a la "comida argentina".

De las diversas características atribuidas a la cocina coreana, la "variedad", en tanto valor positivo ocupa un lugar destacado en los discursos de los migrantes entrevistados. Esta "variedad", además aparece asociada a otros valores, el ser "sana, saludable", y a comensales que favorecen el compartir.

La variedad que caracteriza a la cocina coreana, la cantidad de verduras y los métodos de cocción contribuyen a que sea percibida como "sana y liviana", en oposición a otras cocinas a las que se les atribuyen los valores opuestos. "Es rico y a la vez te sentís bien, te da energía, no te sentís pesado como si te comieras unos ñoquis con estofado" (Clara, 29 años, segunda generación).

En el relato de Oscar, los beneficios de la comida en el plano de la salud, además, están en relación con límites identitarios: la comida coreana es lo que es bueno para los coreanos. Entendemos que esta atribución de propiedades reparadoras para la salud es una forma de conferir significados simbólicos a estas preparaciones. De forma similar a lo que señala Vázquez Medina (2015) en su análisis sobre la alimentación de los migrantes mexicanos en Estados Unidos, estos discursos no solo validan los procesos de totemizacion (Calvo, 1982) o iconización (Janowski, 2013) de ciertos platos, sino que contribuyen a unificar los significados 
asociados a la cocina -y a la cultura- coreanas en un contexto de migración. Además, en este caso, son interesantes los límites identitarios intergeneracionales que plantea, aunque su hijo haya nacido en Argentina es "coreano":

Cuando mi hijo tenía dos años hicimos viaje a Estados Unidos. Fuimos en coche de Los Ángeles a Las Vegas, y él se mareó, vomitó, todo eso. Cuando llegamos a Las Vegas fuimos a un restaurant coreano y comió, comió y se reavivó. A pesar de que nació en Argentina (Oscar, 50 años, generación 1.5).

En cuanto al segundo eje "variedad-compartir", como mencionamos, la comensalidad tradicional coreana se compone de arroz, sopa, banchan y quizás alguna otra preparación caliente asada o estofada. Lo único que se sirve de forma individual es el arroz, y en algunos casos la sopa, mientras que los demás platos son colocados en el centro y cada uno toma su bocado. Esta disposición de los platos, genera una forma de comensalidad e inclusive de disposición corporal en la mesa. El cuerpo no se orienta a un plato individual (Simmel, 1986). Por otro lado, como también mencionamos, esta comensalidad está asociada a una forma de preparar y estructurar la comida. Al ser muchos platos no tiene mucho sentido cocinar para uno solo. Es una mesa pensada para alimentar a varios comensales. Los platos se comparten y se sirven a la vez, a diferencia de la estructura típica occidental que se organiza en diferentes momentos sucesivos -entrada, plato principal y postre-.

Tengo más interacción cuando como comida coreana que cuando como comida no coreana (...). Por ejemplo con una fajita, la fajita me la como yo. Me parece que la comida coreana es como más comunitaria y la comida no coreana es más individual (Milagros, 24 años, segunda generación).

Pero también, esta misma característica puede ser valorada negativamente por algunos miembros de la segunda generación.

Lo que no me gusta de la comida coreana es que metes el palito en todos platitos que compartís con otras personas; si voy con gente conocida está bien, pero cuando tengo que ir así a reuniones con gente que no conozco eso es lo que no me gusta (Marisa, 38 años, generación 1.5).

Con respecto a esta representación de la "comida coreana" como saludable, también es interesante preguntarse hasta qué punto y de qué formas, los migrantes coreanos en Buenos 
Aires están permeados por los discursos que destacan diversos beneficios para la salud de esta tradición alimentaria, que desde la década de 1980 ha desplegado el Estado coreano. Migrantes, que en su mayoría dejaron el país natal antes de que estas políticas se hagan efectivas. En el contexto migratorio, estos discursos son reelaborados por el contacto intercultural. La variedad asociada a la salud es valorada frente a la cocina argentina percibida como pesada y menos saludable.

En el caso de algunos entrevistados, inclusive, aparece directamente asociado un "nosotros""ustedes", a la "cocina coreana" y la "cocina argentina", haciendo de este modo una alusión explícita a la identificación, que atraviesa a las distintas generaciones migrantes:

Nosotros tenemos un menú más amplio que ustedes. Entonces teniendo ese menú más amplio....en mi caso por ejemplo me gusta más. Le doy prioridad a la comida coreana (Miguel, 34 años, generación 1.5).

Me di cuenta que en ese sentido soy bastante coreana. Más que nada en la comida en realidad. Me gusta lo que tiene mucho sabor, me gusta la variedad que hay en la comida coreana. Siento que sin comida coreana no viviría más de tres meses (Nadia, 26 años, segunda generación).

La "comida argentina" por el contrario, aparece como pesada, grasosa, monótona, fácil, rápida, individual. Sucede algo similar a lo señalado por Ray (2004) para el caso de los migrantes indios en Estados Unidos, quien sostiene que estos perfiles de migrantes que ubican a su identidad alimentaria de forma, en cierta medida, opuesta a las de la sociedad mayoritaria, construyen narrativas idealizadas sobre su propio pasado, a partir de este antagonismo. Mientras que la valoración positiva de la "comida coreana" la encontramos en los entrevistados de las distintas generaciones, en el caso de la "comida argentina", las representaciones negativas las encontramos en mayor medida en los entrevistados de la primera generación.

Aquí Argentina lo vi, usan mucho aceite, frita, comida frito y con crema y queso. Pero no engorda tanto. Nosotros comer como ellos, no puede caminar parece (Elena, 64 años, primera generación).

[En la comida coreana] Está muy incorporado el equilibrar lo grasoso, lo fresco; ya el paladar lo necesita...acá hay comidas que son frito con frito. Yo te puedo comer una milanesa con papas fritas, pero no puedo comer todos los días de esa manera...ni ahí. Lo puedo llegar a comer una vez, y por lo general como y no como muchas papas fritas...es 
muy raro...si llego a comer milanesa con papas fritas, como muy pocas papas fritas...suele ser así. Prefiero comer milanesa con ensalada... (Santiago, 33 años, generación 1.5).

En su análisis sobre los hábitos alimentarios de los migrantes bengalíes en Estados Unidos, Ray (2014) sostiene que no hay "una" cocina bengalí, sino múltiples variaciones a partir de diferencias de clase, regionales, religiosas, entre otras. La "cocina bengali", la "cocina bengalíamericana" y la "cocina americana" son categorías relacionales que existen en determinada matriz de relaciones. Una no puede existir sin la otra. Como ocurre de forma más amplia con los sentidos de pertenencia, cada una de estas cocinas se define y cobra sentido en contraste y relación con las otras, en tanto son abstracciones analíticas.

Yo cocino depende de lo que quieran comer, comidas sencillas de acá, igual decimos que es comida sencilla porque comparado con la coreana, acá realmente es muy sencillo (Santiago, 33 años, generación 1.5).

Argentina, ensalada, carne, tarta, muy fácil, ¿no? Chorizo, todo eso. Corea mucho trabajo (Estela, 65 años, primera generación).

Pero así mismo, también encontramos en nuestros entrevistados valoraciones positivas sobre la cocina argentina, sobre todo en torno al plato nacional -y masculino- por excelencia (Álvarez, 2001), la carne vacuna asada.

Yo no avisé, pero cuando llegue acá, una sorpresa que mi amigo me fue a buscar al barco, (...) Me hizo sorprender. ¿El me llevó a dónde? A calle Lavalle, en el centro, 9 de julio, todo teatros, comida, restaurant. Había una casa muy grande de asado, ¿emporio de asado puede ser? Justo ahí había una vidriera grande y jahí estaba parado el asado! ¡Carne! Me llevó allá (Norberto, 77 años, primera generación).

Por otro lado, encontramos en los relatos de los entrevistados la idea de que la "pesadez" de la comida argentina es "cortada" con comida coreana. Esta necesidad, en muchos casos está sustentada en una idea de equilibrio. Así aparecen pares de alimentos especialmente compatibles: asado o carnes con kimchi, "para cortar". O banchan para acompañar milanesas.

Nosotros mucho carne no me gusta, por eso kimchi jjigae limpia (Estela, 65 años, primera generación). 
Generalmente está el kimchi, ya es la costumbre, y si no está el kimchi...bueno, algo similar. Que tenga algo picante...o un sabor....como para cortar. Nosotros le decimos, entre comillas, que es medio pesado, ¿no? Los coreanos dicen que las comidas tienen mucha salsa, o mucho queso, entonces necesitan comer algo picante como para cortar ese gusto. Por eso ahí se usa el kimchi (Nadia, 26 años, segunda generación).

Pastas argentinas le gustan y mezcla un poco el modo coreano y el modo argentino. Le pone queso entonces eso hace que sea un poco pesado, espeso, entonces le pone un poco de picante, ají molido, cosas así para equilibrar. Y también trata de equilibrar toda la carne que se come acá, con un poquito más de verduras que son más coreanas (Luis, 70 años, primera generación).

\section{Notas finales}

A lo largo de este trabajo nos propusimos indagar sobre las representaciones de la cocina coreana entre los migrantes de ese origen en la Ciudad de Buenos Aires, problematizando la construcción de estas tradiciones con anclaje nacional. Desde nuestro enfoque entendemos a la tradición alimentaria en tanto discurso: narración que liga a los procesos sociohistóricos que conforman el sistema ideológico a través del cual se comprende el mundo, con el presente y el futuro. Estos discursos se reconstruyen cotidianamente a través del hacer, del uso, de las prácticas, y se despliegan en distintos espacios sociales. Entre todos ellos, aquí nos concentramos en las políticas estatales.

En el caso coreano, las políticas públicas orientadas a la cocina nacional han atravesado distintos gobiernos, pero tomaron especial fuerza a mediados de la década del 2000 durante un gobierno de centroderecha, encabezado por un líder que armó su carrera en el sector privado, que se presenta a sí mismo como "pro-negocios", e incluso fue CEO de una de las mayores empresas coreanas; en un país con una democracia joven que atravesó en los últimos años profundos cambios económicos, sociales, culturales, políticos e institucionales. Aunque no contamos con la información detallada de los actores intervinientes, podemos sostener que en el diseño de estas políticas participaron sectores gubernamentales, profesionales técnicos y también el sector privado ligado a los alimentos. Las empresas de la industria alimentaria jugaron un importante rol en la configuración de estas políticas, tanto en el planteamiento del problema como en la construcción de sus soluciones. La impronta empresarial atraviesa esta serie de políticas, desde proponerse mejorar la imagen de la "marca Corea", hasta el rol 
asignado a los restaurantes y alimentos industrializados. Entendemos que estos discursos, junto con otros intervinientes en el espacio social, han contribuido a esa construcción que es la tradición alimentaria.

Además, en el contexto migratorio algunos elementos de la trama cultural van a ser idealizados, tomados, utilizados y proyectados en relación con procesos identitarios. Este es el caso de ciertos alimentos y prácticas relacionados con la comida para la comunidad coreana en Buenos Aires. Estas construcciones serán puestas en juego con los particularismos del contexto local.

Encontramos que en este contexto, el referente nacional -"comida coreana"- acentúa su importancia simbólica, que además se re-construye en relación a la cocina argentina. Dentro de las características de la cocina coreana, la "variedad" (que encierra sentidos heterogéneos, seguramente esta idea de variedad será distinta en nuestros entrevistados según su generación y su género, entre otras características) ocupará un lugar destacado en los discursos, y será la marca de cierto gusto asociado a sentidos de pertenencia. La variedad, además, profundiza sus valores positivos en sus relaciones con la salud y una comensalidad que privilegia el grupo y la familia.

Como sostiene Avieli (2005), al ser considerada la comida como una cuestión del ámbito mundano, asociada a lo femenino y los alimentos como algo perecedero, el dominio culinario es percibido como no importante ni amenazante, y por lo tanto menos merecedor de control. Pero paradójicamente, es este carácter lo que facilita en la esfera culinaria la negociación de cuestiones problemáticas, delicadas, o incluso prohibidas para los grupos sociales.

\section{Notas}

(1) Se incluyó a quienes migraron siendo adultos -generación 1.0-, a quienes lo hicieron siendo pequeños, crecieron y fueron criados en la sociedad de destino -generación 1.5-, como también a quienes nacieron en Argentina de padres coreanos -generación 2.0-

(2) La Tesis de Maestría en Investigación en Ciencias Sociales titulada Comida, interculturalidad e identidad. Hábitos alimentarios de los migrantes coreanos en la Ciudad de Buenos Aires.

(3) Siempre que mencionamos a Corea en este artículo nos referimos a la República de Corea (Corea del Sur).

(4) Baek-ku significa 109 en coreano. Este nombre que la comunidad le adjudicó al barrio, hace referencia al ómnibus colectivo- que lo conecta con el centro de la Ciudad. Se concentra en la avenida Carabobo (que cuadras más tarde pasa a llamarse Corea) desde avenida Eva Perón hasta Castañares y hacia los alrededores. La avenida Carabobo es el eje comercial, en tanto que en calles cercanas se instalan las residencias.

(5) Preparación tradicional de la cocina coreana que consiste en el fermento de algún vegetal, más comúnmente repollo akusay, col china o baechu. Según lo define el CODEX, "código alimentario" de la FAO, es un preparado con 
variedades de col china -brassica pekinensis-, en que estas deberán estar exentas de defectos notables, recortadas para eliminar las partes no comestibles, saladas, lavadas con agua potable, y escurridas para eliminar el exceso de agua. Podrán estar o no cortadas en trozos o partes de tamaño idóneo. También incluye una mezcla de condimentos, principalmente pimentón rojo (Capsicum annuum L.), ajo, jengibre, variedades comestibles de Allium distintas del ajo, y rábano. Estos ingredientes podrán estar desmenuzados, rebanados, rotos en trozos. La preparación debe fermentar antes o después de ser envasado en envases apropiados para asegurar la maduración y conservación apropiadas del producto mediante producción de ácido láctico a bajas temperaturas (CODEX STAN 223-2001).

(6) Salsa de soja.

(7) Pasta picante y algo dulce de ají, arroz glutinoso, porotos de soja fermentados y sal.

(8) Pasta de fermento de porotos de soja. Similar al miso japonés.

\section{Bibliografía}

Álvarez, M. (2001). La cocina como patrimonio (in) tangible. Buenos Aires: Comisión para la preservación histórico cultural de la ciudad de Buenos Aires.

Arfuch, L. (comp.) (2002). Identidades, sujetos y subjetividades. Buenos Aires: Prometeo.

Avieli, N. (2005). Vietnamese New Year Rice Cakes: Iconic Festive Dishes and Contested National Identity. Ethnology, 44(2), 167-187.

Barth, F. (1976). Los grupos étnicos y sus fronteras. La organización social de las diferencias culturales. México D.F: Fondo de Cultura Económica.

Calvo, M. (1982). Migration et alimentation. Social Science Information, 21(3), 383-446.

Cho, H. S. (2007). Food and Nationalism: Kimchi and Korean National Identity. The Korean Journal of International Relations, 46(5), 208-228.

Delmonte, R. (2016). Restaurantes coreanos en la Ciudad de Buenos Aires. Comida, cultura e identidad en la diáspora. Sociedade e Cultura, Dossier temático "Comida e Cultura", 18(1), 29-40

Delmonte, R. (2017). Espacios coreanos en la diáspora. Comer en las iglesias cristianas de la comunidad coreana en Buenos Aires. En de la Vega, L. (Comp). Actas VIII Congreso Nacional de ALADAA, Asociación Latinoamericana de Estudios de Asia y África. Lomas de Zamora, Argentina.

Fischler, C. (1995). El omnívoro. Barcelona: Anagrama.

Gimenez, G. (2009). Cultura, identidad y memoria. Materiales para una sociología de los procesos culturales en las franjas fronterizas. Frontera Norte, 21(41), 7-32.

Grimson, A. (2000). El puente que separó dos orillas. En Grimson, A. (ed.). Fronteras, naciones e identidades: la periferia como centro (pp. 201-230). Buenos Aires: CICCUS-La Crujía. 
Grimson, A. (2011). Los límites de la cultura. Crítica de las teorías de la identidad. Buenos Aires: Siglo Veintiuno Editores.

Hall, S. (2011). ¿Quién necesita identidad? En Hall, S. y du Gay, P. (comp). Cuestiones de identidad cultural. Buenos Aires: Amorrortu.

Hobsbawm, E. y Ranger, T. (2002[1983]). La invención de la tradición. Barcelona: Crítica.

Janowski, M. (2012). Consuming Memories of Home in Constructing the Present and Imagining the Future. Food and Foodways: Explorations in the History and Culture of Human Nourishment, 20(3), 175-186.

Medina, X. (2003). Ciudad, etnicidad y alimentación. Restaurantes, tabernas y la construcción de territorios de identidad vascos en Barcelona. Zainak, 24, 835-846.

Mera, C. (2007). Globalización e identidades migrantes. Corea y su diáspora en la Argentina. (Tesis de Doctorado en Ciencias Sociales). Facultad de Ciencias Sociales, Universidad de Buenos Aires.

Mintz, S. W. (1997). Time, Sugar and Sweetness. En Counihan, C. Food and Culture: a Reader. London: Routledge.

Ongkowidjojo (2015). Enhancing competitive identity in global competition: A comparative study of gastrodiplomacy in Malaysia and South Korea. Jurnal Universitas Paramadina, 12(1).

Osetrava, M. (2010). Korean Cuisine and its Role in the Process of National Identity Making in Contemporary South Korea. Korean Studies in Shifted, Proceedings of the 10th Pacific Asian Conference on Korean Studies.

Pettid, M. (2008). Korean cuisine. An illustrated history. Londres: Reaktion Books.

Pham, M. J. (2013). Food as communication: A case study of South's Koreas Gastrodiplomacy. Journal of International Service, 22(1), 1-22.

Ray, K. (2014). The migrant's table. Meals and memories in Bengali-American Households. Philadelphia: Temple University Press.

Ricoeur, P. (1996[1990]). Sí mismo como otro. Mexico D.F.: Siglo XXI Editores.

Santos Luiz Brightwell, M. (2012). A Taste of Home? Food, Identity and Belonging among Brazilians in London. (Thesis submitted to the degree of Ph. D. Department of Geography). Royal Holloway, University of London.

Simmel, G. (1986[1910]). Sociología de la comida. En El individuo y la libertad. Ensayos de crítica de la cultura. Barcelona: Península.

Vázquez Medina, J. A. (2015). De la nostalgia culinaria a la identidad alimentaria transmigratoria: la preparación de alimentos en restaurantes mexicanos en Estados 
Unidos. (Tesis presentada para obtener el Doctorado en Alimentación y nutrición). Universidad de Barcelona.

Walraven, B. C. A. (2002). Bardot soup and Confucian's meat: food and Korean identity in global context. En K. J. Cwiertka \& B. C. A. Walraven (Eds.). Asian food: the global and the local (pp. 95-115). Honolulu: University of Hawai Press.

Young, R. O. (2005). Authenticity and representation: cuisines and identities in KoreanAmerican diaspora. Postcolonial Studies, 8(1), 109-125. 